\title{
Pediatric sepsis screening in US hospitals
}

\author{
Matthew A. Eisenberg ${ }^{1,2 \bowtie}$ and Fran Balamuth ${ }^{3,4,5,6}$ \\ (c) The Author(s), under exclusive licence to the International Pediatric Research Foundation, Inc 2021
}

\begin{abstract}
Sepsis is a major cause of morbidity and mortality in children. While adverse outcomes can be reduced through prompt initiation of sepsis protocols including fluid resuscitation and antibiotics, provision of these therapies relies on clinician recognition of sepsis. Recognition is challenging in children because early signs of shock such as tachycardia and tachypnea have low specificity while hypotension often does not occur until late in the clinical course. This narrative review highlights the important context that has led to the rapid growth of pediatric sepsis screening in the United States. In this review, we (1) describe different screening tools used in US emergency department, inpatient, and intensive care unit settings; (2) highlight details of the design, implementation, and evaluation of specific tools; (3) review the available data on the process of integrating sepsis screening into an overall sepsis quality improvement program and on the effect of these screening tools on patient outcomes; (4) discuss potential harms of sepsis screening including alarm fatigue; and (5) highlight several future directions in sepsis screening, such as novel tools that incorporate artificial intelligence and machine learning methods to augment sepsis identification with the ultimate goal of precision-based approaches to sepsis recognition and treatment.
\end{abstract}

Pediatric Research (2022) 91:351-358; https://doi.org/10.1038/s41390-021-01708-y

\section{IMPACT:}

- This narrative review highlights the context that has led to the rapid growth of pediatric sepsis screening nationally.

- Screening tools used in US emergency department, inpatient, and intensive care unit settings are described in terms of their design, implementation, and clinical performance.

- Limitations and potential harms of these tools are highlighted, as well as future directions that may lead to a more precisionbased approach to sepsis recognition and treatment.

\section{INTRODUCTION}

Sepsis, a life-threatening response to infection with organ dysfunction, is a major cause of morbidity and mortality in children. ${ }^{1-5}$ While adverse outcomes can be reduced through prompt initiation of sepsis protocols including fluid resuscitation and antibiotics, provision of these therapies relies on clinician recognition of sepsis. This recognition is particularly challenging in children due to the low specificity of abnormal vital signs such as fever, tachycardia, and tachypnea for identifying children in the early stages of septic shock. $^{6-9}$

Unlike existing algorithms that risk-stratify children upon likelihood of bacterial infection ${ }^{10}$ or general clinical deterioration $^{11}$, sepsis screening tools specifically focus on differentiating the small number of patients who have or will develop severe sepsis or septic shock from the many children with abnormal vital signs due to uncomplicated infection without associated organ dysfunction. ${ }^{12-14}$ Existing tools vary significantly in the framework they use for sepsis identification, how they interface with clinicians and the electronic health record (EHR), and how they are implemented into clinical workflows. An ideal sepsis screening tool should detect sepsis accurately, with sufficient sensitivity to prevent missed cases but a high enough positive predictive value (PPV) to minimize false positive alerts and the ensuing risk of alarm fatigue. It should also provide an alert early enough in the patient's course to add value to the clinical team, rather than identifying a patient already suspected by the clinician to have sepsis. Finally, it should be easy to use, have minimal inter-rater variability, and incorporate seamlessly into clinical workflows.

This manuscript highlights the important context that has led to the rapid growth of pediatric sepsis screening nationally. In this narrative review, we (1) describe different screening tools used in US emergency department (ED), inpatient, and intensive care unit (ICU) settings; (2) highlight details of design, implementation, and evaluation of specific tools; (3) review the available data on the process of integrating sepsis screening into an overall sepsis quality improvement (QI) program and on the effect of these screening tools on patient outcomes; (4) discuss potential harms of sepsis screening including alarm fatigue; and (5) explore several future directions in sepsis screening. These include developing novel tools that incorporate artificial intelligence/machine learning methods to augment sepsis identification, with the ultimate goal of a precision-based approach to sepsis recognition and treatment.

\footnotetext{
${ }^{1}$ Departments of Pediatrics and Emergency Medicine, Harvard Medical School, Boston, MA, USA. ${ }^{2}$ Division of Emergency Medicine, Boston Children's Hospital, Boston, MA, USA ${ }^{3}$ Department of Pediatrics, University of Pennsylvania Perelman School of Medicine, Philadelphia, PA, USA. ${ }^{4}$ Division of Emergency Medicine, Children's Hospital of Philadelphia,

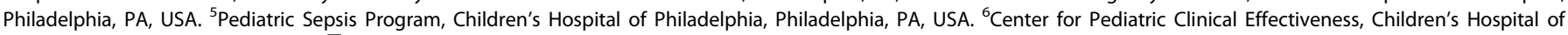
Philadelphia, Philadelphia, PA, USA. ${ }^{\bowtie}$ email: matthew.eisenberg@childrens.harvard.edu
}

Received: 12 April 2021 Revised: 28 July 2021 Accepted: 4 August 2021

Published online: 20 August 2021 


\section{GUIDELINES AND LEGISLATION}

While efforts at systematic screening of children for sepsis date back more than a decade, much of the recent impetus for screening can be traced to the case of Rory Staunton, a 12-yearold New York boy who died of septic shock caused by Group A Streptococcus in 2012. ${ }^{15}$ Rory's death received considerable publicity nationally and led his family to launch an advocacy campaign to increase awareness of sepsis and improve sepsis recognition and treatment in pediatric care. The culmination of this effort was the 2013 passage by the New York state legislature of Rory's Regulations, which required all New York hospitals to implement evidence-based protocols to facilitate early recognition of sepsis through screening tools, identify individuals who qualify for sepsis care, and ensure they receive a care bundle consisting of fluid resuscitation, intravenous (IV) antibiotics, and a blood culture sample. $^{16}$

Due in part to the success of Rory's Regulations, ${ }^{6}$ other states enacted similar protocols. In 2016, Illinois passed Gabby's law, named after 5-year-old Gabrielle Calbo who died of sepsis in 2012. The law requires Illinois hospitals to implement evidence-based guidelines to facilitate rapid recognition and treatment of adults and children with sepsis, train staff on sepsis treatment and identification, and report sepsis data. ${ }^{17}$ Several other states are considering similar legislation or have sponsored QI initiatives aimed at improving sepsis identification and treatment within their hospitals. ${ }^{18}$ Most recently, federal legislation based on Gabby's law has been proposed that would mandate evidencebased sepsis identification and treatment protocols for all hospitals in the United States as a condition of Medicare enrollment. ${ }^{19}$

Along with legislative mandates, pediatric sepsis screening has been buoyed by the recommendations of professional organizations. In 2017, the American College of Critical Care Medicine recommended routine pediatric sepsis screening as part of its Clinical Practice Parameters for Hemodynamic Support of Pediatric and Neonatal Septic Shock. ${ }^{20}$ Specifically the national practice guideline recommends that each institution implement sepsis care bundles for children that include a "recognition bundle containing a trigger tool for rapid identification of patients with suspected septic shock at that institution." This was followed in 2020 by the recommendation of the Surviving Sepsis Campaign that hospitals implement "systematic screening for timely recognition of septic shock and other sepsis-associated organ dysfunction" in acutely unwell children, though this recommendation was noted to be weak and based on a low quality of evidence. $^{21}$

\section{TYPES OF SCREENING}

Given this pressure from regulators, legislatures, and professional societies, as well as increasing recognition of the value of sepsis QI programs, ${ }^{8,22,23}$ sepsis screening has proliferated in US pediatric acute care settings in the past decade. However, the tools employed vary significantly in terms of their parameters and form, with little consensus as to which tool best identifies children at high risk of septic shock while also minimizing false positive alerts. Additionally, reports of these tools in the medical literature have used different criterion standards for sepsis, making direct comparison of their performance difficult. Here we review the differences in sepsis screening tools that have been reported in the literature.

\section{Framework for sepsis identification}

While all sepsis screening tools rely on combinations of abnormal vital signs and physical exam findings in the presence of suspected infection, there are substantial differences between them depending on the framework they utilize for sepsis prediction and/or identification. Tools such as the Pediatric Septic Shock Collaborative's (PSSC) sepsis trigger tool rely on abnormal vital signs and physical exam findings as well as the presence of medical conditions that confer increased risk of sepsis and in so doing aim to identify patients prior to the onset of septic shock or severe sepsis. ${ }^{24}$ Other screening tools utilize a framework for defining sepsis, such as the systemic inflammatory response syndrome (SIRS)/sepsis/severe sepsis definitions set out by the International Pediatric Sepsis Consensus Conference (Table 1). ${ }^{25}$ Some researchers created homegrown models of sepsis prediction, then used those models to create de novo screening tools. ${ }^{12,26}$ As a result, the various screening tools differ not just in which parameters they utilize but how they define the thresholds at which those parameters signify increased sepsis risk.

\section{Form of tool}

Even tools that share frameworks of sepsis identification can vary significantly in their form, how they are utilized clinically, and how they are incorporated into the EHR. In so doing, identical sets of criteria can actually have different clinical performance. The earliest screening tools, such as the PSSC trigger tool, were paper forms designed to be completed at the time of ED triage. ${ }^{24}$ After such tools are embedded in practice, the next step is usually incorporation into the EHR in electronic form. ${ }^{27}$ In addition to better integration into electronic documentation, EHR-embedded tools facilitate repeated screening, rather than only at the time of triage. Such repeat assessments can be triggered by clinician concern or by the patient meeting some other objective criteria, such as new onset of fever or a higher Pediatric Early Warning Score. ${ }^{11}$ Leveraging the learning health system, more recent automated tools continuously incorporate new data from the patient's EHR and can therefore alert at any time in an encounter that pre-specified criteria are met. ${ }^{12,14}$

\section{Population screened}

A final difference in available screening tools is in the population screened. Some tools are utilized as part of a broad screening strategy for all patients. Other tools are designed for a more targeted approach-for instance, only those children with suspected infection or only those who have abnormal vital signs or other concerning clinical findings. The more targeted approaches have the advantage of fewer false positive alerts (since sicker patient groups will have a higher sepsis prevalence),

Table 1. Summary of International Pediatric Sepsis Consensus Conference definitions. ${ }^{23}$

\begin{tabular}{ll} 
SIRS & $\begin{array}{l}\text { Abnormal values for at least two of the following, one of which must be temperature or leukocyte count } \\
\text { - Temperature } \\
\text { - Leukocyte count } \\
\text { - Heart rate } \\
\text { - Respiratory rate }\end{array}$ \\
& SIRS in the presence of a suspected or proven infection \\
Sepsis & Sepsis plus cardiovascular organ dysfunction and/or acute respiratory distress syndrome and/or two or more organ dysfunctions \\
Severe sepsis $\quad$ Sepsis and cardiovascular organ dysfunction \\
\hline Septic shock $\quad$ S
\end{tabular}


but at the risk of missing sepsis cases in patients in whom the provider has not already identified a higher severity of illness.

\section{AVAILABLE SCREENING TOOLS ED-based tools}

While sepsis screening tools are utilized in clinical practice settings as varied as ambulatory clinics and intensive care, the majority of reports of sepsis screening in the medical literature originate in the ED.

The first report of systemic screening of children in the ED for sepsis came from Texas Children's Hospital in 2012 in the form of an electronic, triage-based "Best Practice Alert (BPA).."13,28 The BPA was designed to calculate a heart rate corrected for pyrexia by adjusting the measured heart rate down 5 beats per minute for every $1^{\circ} \mathrm{C}$ in temperature elevation and comparing that to ageappropriate normal values. When the heart rate was outside of the normal range, the BPA displayed a prompt alert for the triage nurse to ask about medical conditions that rendered a higher risk of sepsis. If positive, or if the child was ill-appearing, the patient would be transferred to a room for immediate evaluation and resuscitation as needed. While the tool was designed for use at triage, it could be repeated at any time during the ED stay. The tool proved to be sensitive in the prediction of sepsis (81\%) but with a low PPV (4\%), reflecting a low specificity and relatively low incidence of sepsis in the population screened. ${ }^{28}$

2012 also saw the creation of the PSSC, a joint QI initiative sponsored by the American Academy of Pediatrics (AAP) and the Children's Hospital Association (CHA), which aimed to reduce sepsis mortality across its member institutions. ${ }^{24,29}$ As part of phase 3 of the initiative, the PSSC created and distributed a septic shock identification tool (Fig. 1). This paper-based tool used a combination of vital signs, physical exam findings, and patient medical history to generate a score for each patient triaged. High-risk patients with a score of $\geq 2$ or any patient with a score of $\geq 3$ were considered to have a positive screen. The PSSC tool was widely disseminated and easily adapted for use in a variety of children's hospitals, with a sensitivity of $99 \%$ and PPV $20 \%$ in one report of its use at Primary Children's Hospital in Salt Lake City, Utah. ${ }^{22}$

While the original PSSC tool was on paper, one of its advantages was easy adaptation into electronic form, as reported by a team at Nationwide Children's in Ohio. ${ }^{27}$ This strategy began with the PSSC identification tool, was then incorporated into the EHR, and was automated to alert when any tool criteria fell outside of the normal range. At the same time, the team made modifications to improve its specificity such as linking it to a triage field that queried for the presence of infection or recent fever, adjusting the respiratory rate values from the original tool and requiring a score of 4 rather than a score of 3 to generate an alert.

A novel approach from Children's Hospital of Philadelphia (CHOP) began by retrospectively validating the performance of the PSSC tool when compared to clinician gestalt, demonstrating that the screening tool was more sensitive $(92.1 \mathrm{vs.} 72.7 \%)$ but less specific (83.4 vs. 99.5\%). ${ }^{30}$ This led the investigators to develop and implement a two-stage identification method that differed from the PSSC tool in terms of the order of items evaluated. The first stage of the alert involved automated surveillance for the presence of bradycardia, tachycardia, or hypotension, which, if present, would trigger a BPA that queried for the presence of fever, hypothermia, or possible infection. ${ }^{31}$ If any of these were present, the nurse would fill out a secondary sepsis screen that asked about capillary refill, mental status, or history of a high-risk condition (Fig. 2a). A positive secondary screen mandated a bedside sepsis huddle by the treating team (Fig. 2b). Additional alerts were built to remind the team to repeat a huddle on patients who are deemed sepsis "watchers"-patients who triggered an alert but did not need immediate resuscitationafter $60 \mathrm{~min}$ (Fig. 2c) and if hypotension develops on a patient with a previous negative alert or huddle (Fig. 2d). The two-stage alert had both high sensitivity (86.2\%) and specificity $(99.1 \%)$; when combined with clinician gestalt, sensitivity improved to $99.4 \%$ without any change in specificity.

The first attempt at a fully automated pediatric sepsis prediction tool was reported by Sepanski and colleagues in 2014, derived from a combined cohort of children presenting to EDs in Memphis, Tennessee and Norfolk, Virginia. ${ }^{12}$ While the algorithm they employed was based on the International Pediatric Sepsis Consensus Conference framework, ${ }^{25}$ they first analyzed the vital signs from $>140,000$ ED encounters to derive new age-specific heart rate and respiratory rate values that constituted their normal range. They then went through an iterative process of comparing patient results to a defined cohort with sepsis until they arrived at a final version of the tool with a sensitivity of $97 \%$ and PPV of $49 \%$.

A similar approach was utilized at Boston Children's Hospital and ultimately put into clinical practice in 2018, using the SIRS/ sepsis/severe sepsis framework with adjusted respiratory rate parameters to create an algorithm that continuously surveilled ED patients for sepsis. The algorithm generated a pop-up alert to all providers caring for the patient if pre-specified criteria were met (Fig. 3a). ${ }^{14}$ When deployed in that ED, the tool was both sensitive $(84.6 \%)$ and specific (91.1\%), though the PPV was just $3.7 \%$, reflecting low sepsis prevalence due to having screened all ED patients, not just those with fever or suspected infection. ${ }^{32}$ To improve on the PPV of the screening algorithm, the authors created tiered alerts of increasing severity (termed SIRS, sepsis, and severe sepsis). As was done in Philadelphia, for patients with a SIRS or sepsis alert, the nurse caring for the patient was prompted to fill out a secondary screening form that queried for the presence of suspected infection, altered mental status, or altered pulses/perfusion (Fig. 3b). A patient was only considered to have a positive sepsis screen if this secondary screen was positive. However, all patients with a severe sepsis alert, triggered by the presence of either cardiac dysfunction or two dysfunctional organ systems, were considered to be at high risk of sepsis and required an urgent sepsis huddle.

A summary of the characteristics of these ED-based sepsis screening tools is shown in Table 2.

\section{Challenges in interpreting screening tool performance}

A number of limitations exist in all of the aforementioned reports of sepsis screening tool performance. First, it is important to note the reference standard against which a screening algorithm is applied when evaluating sepsis screening tools. Clinical definitions of sepsis used in the medical literature vary significantly, from those based on provider actions ("intention to treat sepsis" or use of a sepsis pathway) to use of diagnosis codes to comparison to published clinical criteria. As a result, it is difficult to directly compare the test characteristics of one reported tool to another unless the same reference standard was used and cases identified in a similar manner. Generally, reported test characteristics of tools that used a combination of existing hospital tracking systems and systematic medical record review to identify patients who may have been missed by the tool ${ }^{22,31,32}$ are likely more accurate than those that were derived solely from diagnostic codes for sepsis or tools that were not compared against a sepsis reference standard.

An additional challenge in evaluating pediatric screening tools is the difficulty of accurately identifying false positives. It is impossible to distinguish with current tools whether a child with a positive sepsis alert who received IV fluids and antibiotics and never developed organ dysfunction is a "false positive" who was unnecessarily treated or a "great catch" who would have gone on to develop severe sepsis or septic shock without those interventions. Additionally, alerts that identify a patient clinically deteriorating from an illness other than sepsis may count as "false positives" but actually have considerable value if they help to identify that deterioration and facilitate an intervention. 
PEDIATRIC SEPTIC SHOCK COLLABORATIVE TRIAGE TRIGGER TOOL

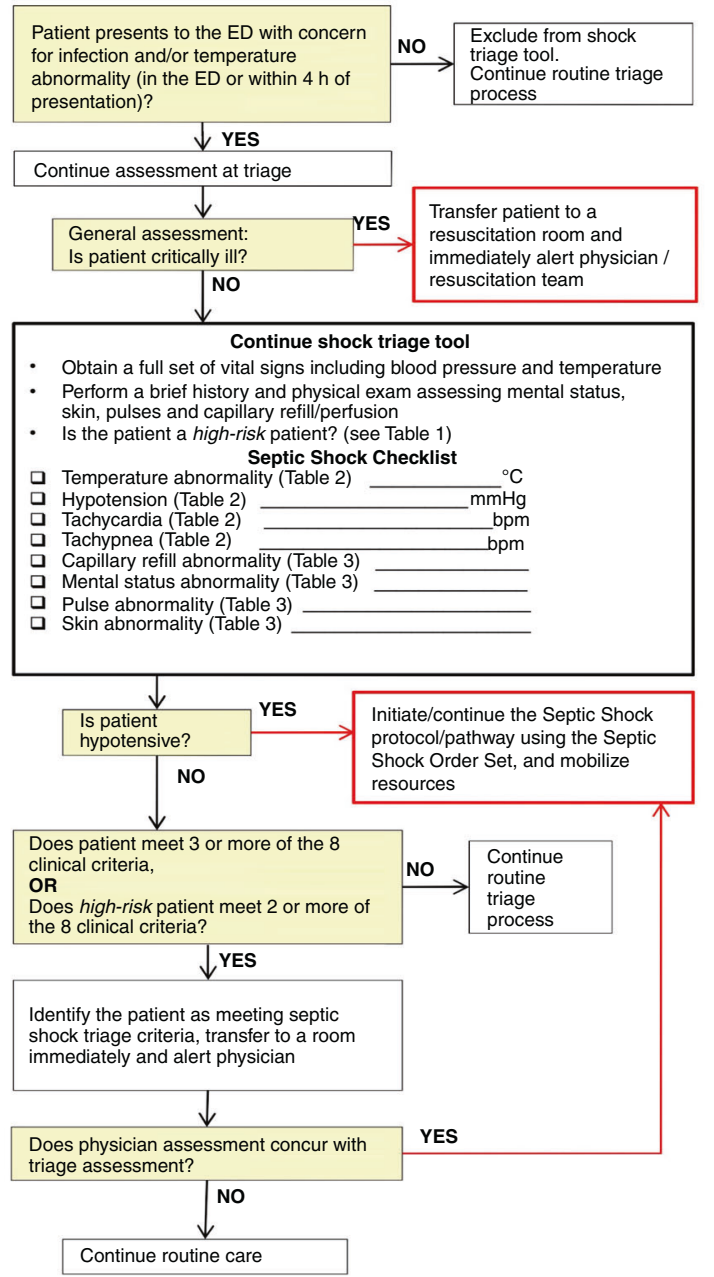

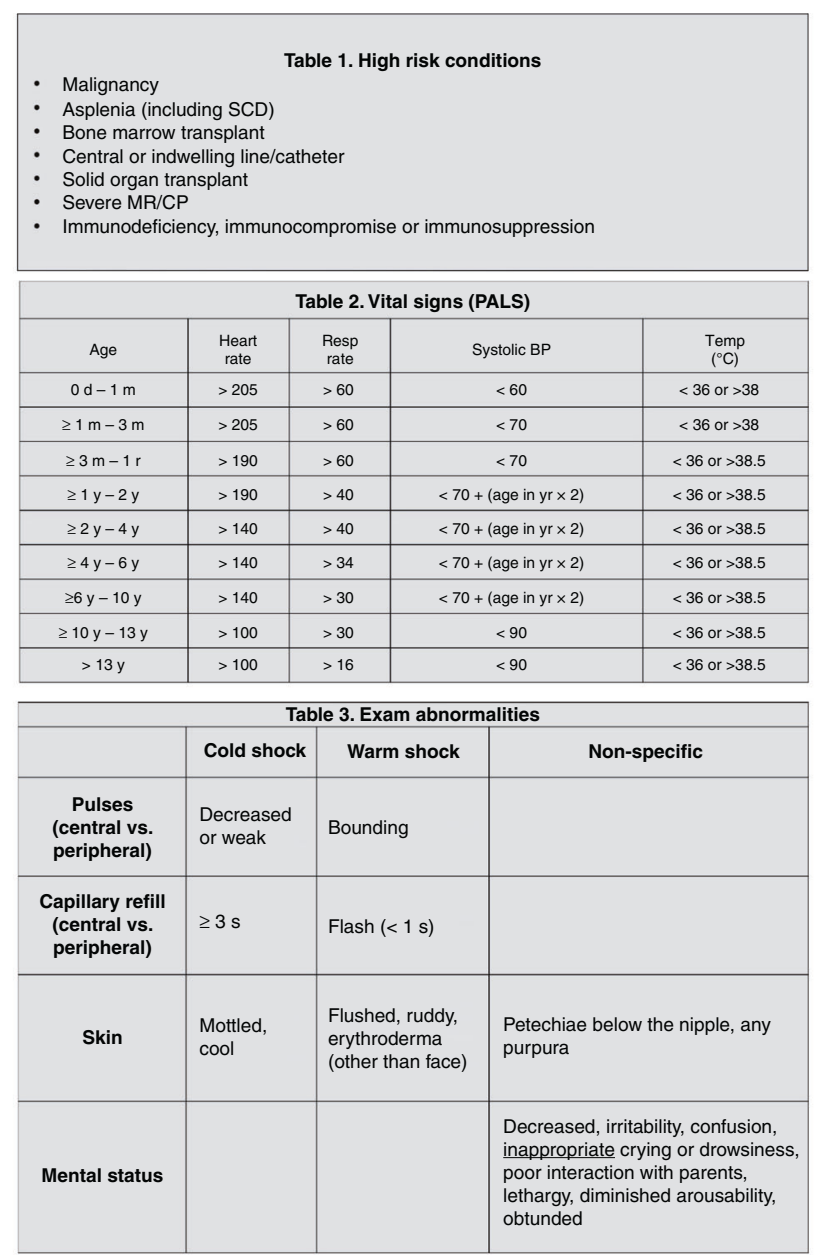

Fig. 1 The pediatric septic shock collaborative triage trigger tool. Sepsis screening tool developed by the Pediatric Septic Shock Collaborative, designed to be used at ED triage on patients with fever or suspected infection.

It is important to note that all of the above reports are single center. As such, it is unclear whether even the best-performing tools do as well at sepsis detection when used outside of the reporting hospital or in other care settings, such as a general ED. In addition, none of the tools described here were evaluated in a randomized fashion, which would help to account for unmeasured confounders. Future studies using adjusted time series analysis or other randomized methods of measuring implementation would help to address this issue.

\section{Sepsis screening outside of the ED}

Sepsis screening outside of the ED has been less frequently reported in the literature, though its use in these settings is increasing in clinical practice. Notably, these tools have generally employed different frameworks for sepsis detection than the EDbased tools, given different patient characteristics and sepsis prevalence in the inpatient and ICU settings. Inpatient tools have the benefit of longer observation times, which allows for utilization of changes or trends in vital signs or other clinical parameters to drive performance. Longitudinal evaluation, however, is a double-edged sword, as issues such as lock out time (i.e., the time after a given alert when additional alerts will no longer fire) following a positive alert must also be addressed. Additionally, while heterogeneous definitions of "time 0 " in the ED can make benchmarking a challenge, it is even more difficult in the inpatient setting where specific time markers such as ED arrival or triage time cannot be used as a proxy for sepsis onset.

In response to Rory's regulations, a team at New York University created a sepsis identification process for their inpatient pediatric unit consisting of a temperature-adjusted vital sign screen followed by physician examination. ${ }^{33}$ The tool identified 38/39 patients with possible sepsis/septic shock (sensitivity $97.4 \%$ ) with PPV of $23.5 \%$ for possible sepsis, though only $3.7 \%$ for severe sepsis/septic shock.

Sepsis screening in the ICU entails a very different set of challenges, as sepsis itself is more common than in the ED or inpatient unit but so are a wide variety of other conditions that may mimic sepsis. A team at Cincinnati Children's created a computerized sepsis alert that was triggered by the presence of abnormal temperature and an order for a blood culture in the prior $6 \mathrm{~h}^{34}$ When present, an interruptive alert would prompt nursing staff to assess the patient's blood pressure, perfusion, and mental status. The alert identified 392/436 patients in the ICU with sepsis (sensitivity $92.5 \%$ ) while maintaining a PPV of $46 \%$.

\section{EFFECT ON PATIENT OUTCOMES}

Many of the available screening tools have proven to be sensitive, specific, and well integrated into clinical practice. In order to lead to better clinical outcomes though, the tool must also show that it 
a

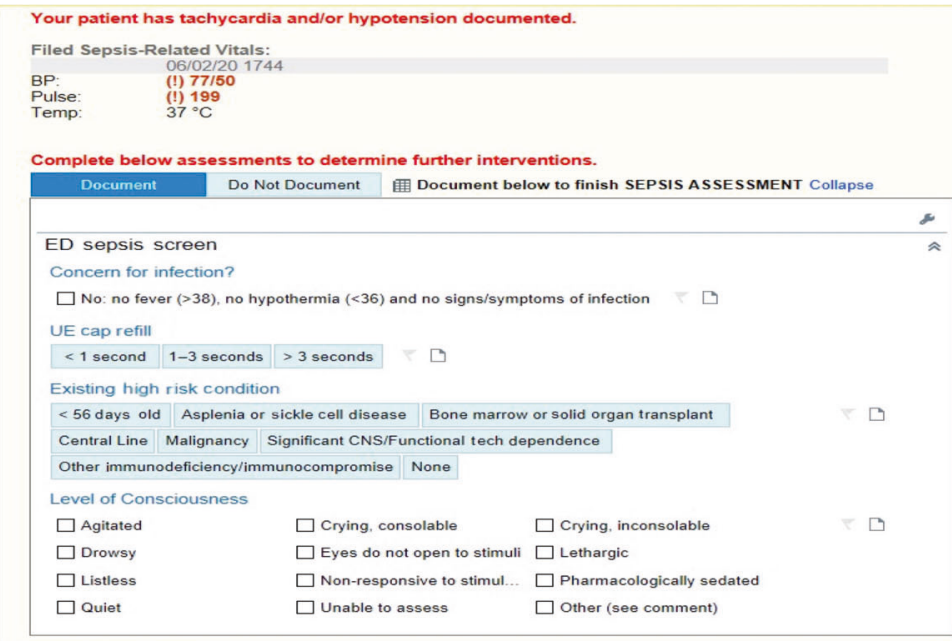

C

\begin{tabular}{l} 
(1) Alerts (1) \\
Impending \\
(1) Sepsis Watcher Patient Identified: Re-Huddle Required $01 \mathrm{~m}$ \\
\hline \$ Sepsis Huddle Results \\
\hline
\end{tabular}

b

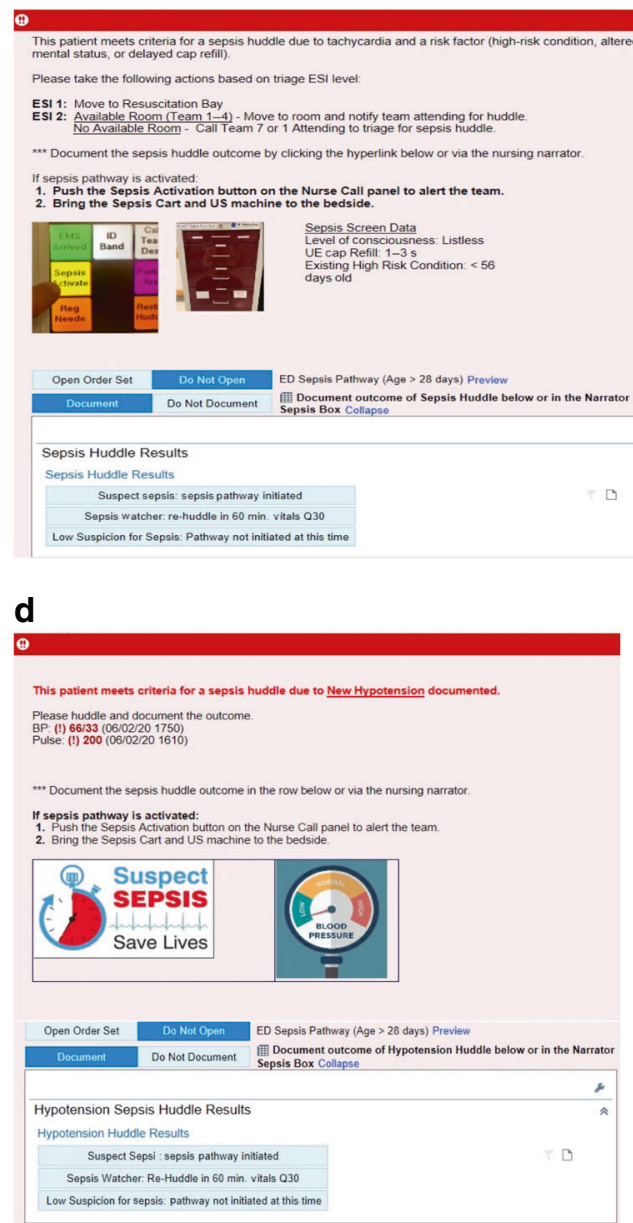

Fig. 2 Screen shots of the Children's Hospital of Philadelphia sepsis alert. Screen shots of the Children's Hospital of Philadelphia sepsis alert: a first-stage alert appears if tachycardia or hypotension is present. The nurse documents concern for infection and any present perfusion abnormality or high-risk condition. Mental status is pre-populated with current documentation, which the nurse may update. b Second-stage alert appears if patient meets criteria for a sepsis huddle. Nurse and either attending or fellow huddle using the sepsis alert response algorithm. c If the patient is deemed a "sepsis watcher," EPIC narrator alert reminds of need to re-huddle in 60 min. $\mathbf{d}$ If new hypotension is noted after prior negative alert or huddle, additional hypotension huddle alert will appear.

leads to improved sepsis recognition when compared to clinician gestalt. Only the tool in use in the CHOP ED showed a decrease in missed sepsis cases after implementation, from $17.3 \%$ of all patients with severe sepsis within $24 \mathrm{~h}$ of ED disposition to $3.8 \%$ after onset of sepsis screening. ${ }^{31}$

Even less clear are the effects of sepsis screening on clinical outcomes, such as organ dysfunction, ICU and hospital length of stay (LOS), ventilator or vasopressor days, and mortality.

When screening tool deployment has been associated with improvement in these outcomes, it has always been as part of a systematic QI program, making it impossible to isolate the effect of the screening tool. At Texas Children's Hospital, for example, Arikan and colleagues reported an impressive $46 \%$ reduction in acute kidney injury as well as shorter hospital and ICU LOS and improved mortality after introduction of a protocol-driven sepsis bundle that included a sepsis recognition tool. ${ }^{35}$ However, the bundle also included successful efforts to improve time to antibiotics and fluid administration and volume of fluid administered, interventions that have previously been strongly linked to improved sepsis outcomes. Similarly, in an analysis by Balamuth and colleagues, bundled sepsis care was associated with decreased duration of organ dysfunction, a difference which remained even after adjustment for timely sepsis therapies. ${ }^{36}$ Conversely, when Eisenberg et al. looked specifically at whether sepsis outcomes improved after implementation of an automated screening tool in their ED, they found no differences in hospital or ICU LOS or mortality. ${ }^{37}$ The improved clinical outcomes reported with bundled sepsis care, but not with sepsis screening alone, suggest that there may be additional benefit from human factors that are elements of bundled care, such as a shared mental model among clinical team members.

\section{IMPLEMENTING SEPSIS SCREENING}

While the effect of sepsis screening alone on patient outcomes may be uncertain, it is well established in the literature that comprehensive sepsis QI programs that include sepsis identification processes can save lives. ${ }^{8,13,35}$ This finding is intuitive-after all, sepsis identification is merely the first step in a process that must then lead to rapid and appropriate provision of fluids, antibiotics, vasoactive infusions, and other critical care interventions. At each step along the way, QI efforts can improve the care provided: a bedside huddle allows for rapid resuscitation when appropriate (and withholding of resuscitation when it is not); use of standardized order sets facilitates ordering recommended antibiotics, pressors, and fluids; skilled nursing and pharmacy care get those medications to the bedside and safely deliver them as quickly as possible; and critical care infrastructure allows for support for failing organ systems. A robust educational 


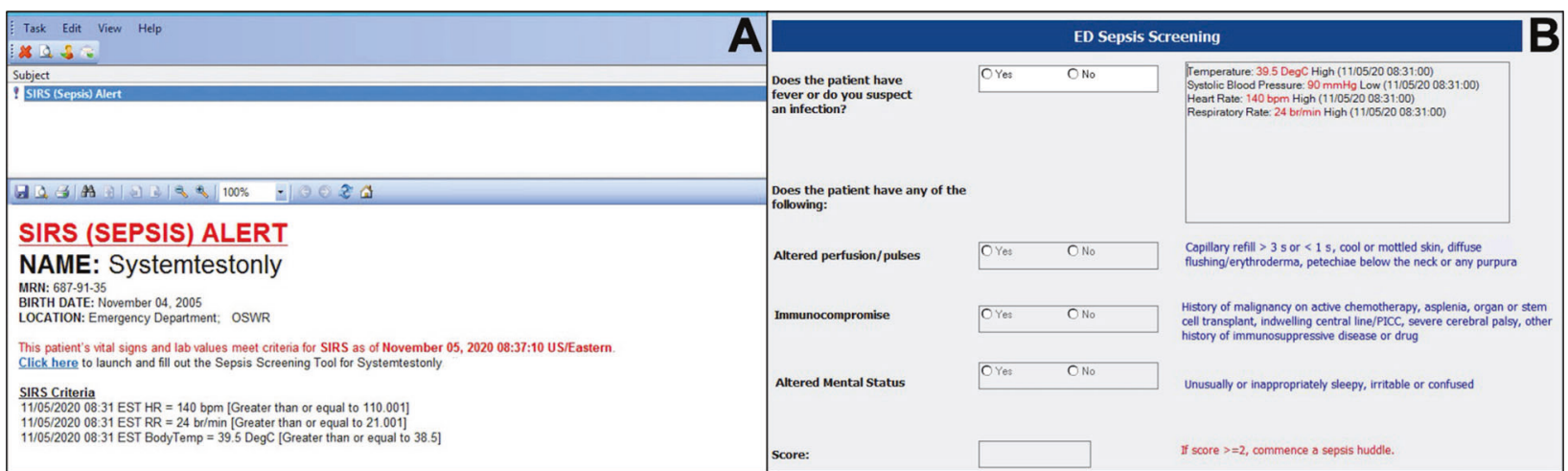

Fig. 3 Screenshots of the Boston Children's Hospital automated sepsis alert. a pop-up alert generated when patient meets pre-specified criteria is sent to all providers who have signed up for the patient on the tracking board and (b) secondary screening form is completed by the nurse for patients with a positive SIRS or sepsis alert. A positive secondary screen, or a "severe sepsis" alert are followed by a sepsis huddle. Icons associated with each alert also appear next to the patient's name on the tracking board.

curriculum supports each of these interventions by continually reinforcing best practices, and tracking of process, outcome, and balancing measures allows education and interventions to be targeted when and where they are needed most.

It is therefore natural that sepsis screening has been an important component of local, state, and national QI efforts. These QI efforts may be mandated by legislation, as in the case of New York and Illinois, or optional collaboratives such as those organized by the AAP and CHA. In New York, Rory's regulations were associated with a 0.59 odds ratio of mortality compared to patients who did not receive the care bundle within an hour. ${ }^{6}$ While the results of the large, national Improving Pediatric Sepsis Outcomes collaborative run by CHA are not yet known, it has $>50$ hospitals enrolled with an aim of reducing sepsis-attributable mortality by $25 \%$ through interventions that start with widespread utilization of sepsis screening. ${ }^{38}$

Importantly, all reports of sepsis screening in the medical literature come from large pediatric referral centers. Yet, as the Surviving Sepsis Campaign reminds us, sepsis screening tools must be "adapted to the type of patients, resources, and processes within each institution." 21 Thus, the dearth of data from outside of tertiary pediatric care centers is concerning but presents opportunity. It is concerning because most children in the US present to general ED's with low volumes of pediatric sepsis, ${ }^{39}$ a setting in which virtually nothing is known about the effect of sepsis identification efforts. The opportunity exists because what little we do know suggests that sepsis Ql efforts, including sepsis screening, are likely to be more effective in hospitals that infrequently care for septic children than in large pediatric centers, and adoption of best practices in those settings may therefore have a large impact nationally on pediatric sepsis outcomes. ${ }^{40}$

\section{POTENTIAL FOR HARM}

Despite the potential advantages of sepsis screening in earlier identification and treatment of pediatric severe sepsis, skeptics abound. Partially, this is due to the lack of proven impact on patient outcomes. There are also concerns about the unintended effects of sepsis screening -in particular potential for alarm fatigue, inappropriate resource utilization, and over-reliance on screening. ${ }^{41}$ It is critical that studies assessing sepsis screening tool performance also examine these balancing measures to ensure that any potential negative effects are mitigated.

Alarm fatigue, in which a high frequency of false positive alarms leads to clinician desensitization and subsequent failure to respond to true alarms, has been shown to have a deleterious effect in a wide variety of patient care settings. ${ }^{42,43}$ Alarm fatigue is largely a function of PPV - if a high proportion of alarms are "true positives," clinicians are unlikely to ignore them. As the proportion of false alarms increases, so does the risk of alarm fatigue. ${ }^{44,45}$ One study of adults showed that the introduction of an automated sepsis alert with a low PPV did in fact lead to alarm fatigue and worse patient outcomes. ${ }^{46}$ It is reasonable to assume that this risk exists in pediatric sepsis as well, and it is therefore paramount that screens have sufficiently high PPV to prevent this outcome.

Of course, a high number of false positive alerts may not just affect those children with sepsis, but those without it. Potential adverse effects on the child with a false positive sepsis screen include unnecessary placement of venous catheters and inappropriate administration of IV antibiotics and IV fluid. The health care system itself may also be impacted by unnecessary hospital or ICU admission for children who would not otherwise have required such an admission without the concerns raised by a false positive sepsis screen. While the fear of these potential harms are legitimate, they have not been demonstrated in the medical literature. In fact, the only study that specifically examined this question showed that there was no change in administration of IV antibiotics or IV fluid, ED LOS, or hospital admission among patients with a false positive sepsis screen in a pediatric ED after implementation of automated sepsis screening. ${ }^{47}$

A final concern that has been raised on systematic sepsis screening is that, once clinicians learn to rely on such screens, they may fail to identify patients who have sepsis but did not trigger an alert or positive screen. Such false negatives can, of course, be prevented by creating a screen that is sufficiently sensitive for detecting sepsis early in the ED or hospital stay, but no such screen will ever identify all sepsis cases. However, the available literature ${ }^{30,31}$ suggests that, if care for septic children is sometimes delayed by false negative sepsis screening, such cases are significantly outnumbered by the cases of sepsis identified by the screen that would have otherwise been missed. Whether this still holds true in general EDs, which have a lower sepsis prevalence than the pediatric EDs from which all of these published reports have originated, should be a priority in future research.

\section{FUTURE DIRECTIONS}

Machine learning and artificial intelligence offer the promise of creating better sepsis identification tools that leverage big data and can incorporate elements from the EHR that previously required manual input. Rather than utilize rule-based thresholds such as SIRS, the pediatric early warning score, or the sequential organ failure assessment, ${ }^{48}$ such algorithms are trained to run complex tasks on large amounts of data in order to predict adverse outcomes. This also allows integration of dynamic vital sign changes, which are likely to be more sensitive in detecting 
alterations in a patient's clinical status than absolute values, particularly in patients whose baseline vital signs or laboratory values are abnormal. Machine learning may therefore result in prediction rules that detect sepsis both more accurately and earlier than rule-based thresholds. Machine learning has been reported to improve sepsis identification among adults ${ }^{49,50}$ but is still in its nascent stages in pediatrics. ${ }^{51}$ In addition, biomarkers, both known and novel, may ultimately add important information to allow prognostic and predictive enrichment to facilitate decision making in children with sepsis and opens the possibility of precision-based therapies.

Perhaps equally important to the set of criteria that trigger an alert is the integration of that alert into the real-world clinical environment. Alerts that are poorly integrated into EHR workflow are at risk of being ignored and may worsen alarm fatigue. Human factors principles provide a promising avenue forward. Human factors engineering applies knowledge about human capabilities and limitations to the design of products, processes, systems, and work environments. ${ }^{52}$ These concepts can be applied to create sepsis alerts that better translate available demographic and clinical data into usable information that supplements available data rather than distracts from it and makes it easier to provide recommended treatment at the bedside. ${ }^{53}$ Ideally, by combining machine learning that better predicts development of septic shock with human factors principles that lead to better EHR integration, the future of sepsis prediction is earlier identification of those at risk with actionable clinical decision support that facilitates giving the right care at the right time to the right patient.

\section{CONCLUSIONS}

Many questions remain about the optimal sepsis screen for children. It is unclear what clinical criteria best identify children with sepsis while minimizing risk of false positive alerts. Likewise, both automated and manual sepsis screening have relative advantages and disadvantages, and there is no consensus on whether either one or a hybrid of the two performs best. Not surprisingly, the Surviving Sepsis Campaign guidelines identified QI screening tool algorithms to recognize clinical deterioration as a knowledge gap in sepsis pathophysiology and need for clinical trials on pediatric sepsis recognition as one of its key research opportunities. ${ }^{21}$

The answers to these questions are likely to be context specific-a tool that performs well in an ED may be insufficiently specific for use in an ICU setting. At the same time, a community ED remote from tertiary or ICU care may prefer a less specific but more sensitive tool or one that values earlier alerting over accuracy in order to facilitate transfer of children prior to clinical deterioration. To answer these questions, multi-center studies will be needed across a spectrum of clinical settings to overcome the relatively low sepsis incidence at any given site, and machine learning will need to be leveraged to best make use of big data.

What is clear is that systematic sepsis screening of children can help augment recognition by clinicians in the ED, inpatient, and ICU settings and, as part of a comprehensive QI program, improve patient outcomes.

\section{REFERENCES}

1. Balamuth, F. et al. Pediatric severe sepsis in U.S. children's hospitals. Pediatr. Crit. Care Med. 15, 798-805 (2014).

2. Odetola, F. O., Gebremariam, A. \& Freed, G. L. Patient and hospital correlates of clinical outcomes and resource utilization in severe pediatric sepsis. Pediatrics https://doi.org/10.1542/peds.2006-2353 (2007).

3. Ruth, A. et al. Pediatric severe sepsis: current trends and outcomes from the pediatric health information systems database. Pediatr. Crit. Care Med. https://doi. org/10.1097/PCC.0000000000000254 (2014).

4. Schlapbach, L. J. et al. Mortality related to invasive infections, sepsis, and septic shock in critically ill children in Australia and New Zealand, 2002-13: a multicentre retrospective cohort study. Lancet Infect. Dis. 15, 46-54 (2015). 
5. Weiss, S. L. et al. Global epidemiology of pediatric severe sepsis: the sepsis prevalence, outcomes, and therapies study. Am. J. Respir. Crit. Care Med. 191, 1147-1157 (2015).

6. Evans, I. V. R. et al. Association between the new york sepsis care mandate and inhospital mortality for pediatric sepsis. JAMA 320, 358 (2018).

7. Weiss, S. L. et al. Delayed antimicrobial therapy increases mortality and organ dysfunction duration in pediatric sepsis. Crit. Care Med. 42, 2409-2417 (2014).

8. Paul, R., Neuman, M. I., Monuteaux, M. C. \& Melendez, E. Adherence to PALS sepsis guidelines and hospital length of stay. Pediatrics 130, e273-e280 (2012).

9. Scott, H. F., Deakyne, S. J., Woods, J. M. \& Bajaj, L. The prevalence and diagnostic utility of systemic inflammatory response syndrome vital signs in a pediatric emergency department. Acad. Emerg. Med. 22, 381-389 (2015).

10. Kupperman, N. et al. A clinical prediction rule to identify febrile infants 60 days and younger at low risk for serious bacterial infections. JAMA Pediatr. 173, 342-351 (2019).

11. Duncan, H., Hutchison, J. \& Parshuram, C. S. The pediatric early warning system score: a severity of illness score to predict urgent medical need in hospitalized children. J. Crit. Care 21, 271-278 (2006).

12. Sepanski, R. J. et al. Designing a pediatric severe sepsis screening tool. Front. Pediatr. 2, 56 (2014).

13. Cruz, A. T. et al. Implementation of goal-directed therapy for children with suspected sepsis in the emergency department. Pediatrics 127, e758-e766 (2011).

14. Eisenberg, M., Madden, K., Christianson, J. R., Melendez, E. \& Harper, M. B. Performance of an automated screening algorithm for early detection of pediatric severe sepsis. Pediatr. Crit. Care Med. https://doi.org/10.1097/pcc.0000000000002101 (2019).

15. Dwyer, J. An infection, unnoticed, turns unstoppable. The New York Times (11 July 2012).

16. New York State Law: Title 10 of the New York State Codes, Rules and Regulations (Sections 405.2 and 405.4). https://www.health.ny.gov/facilities/ public_health_and_health_planning_council/meetings/2013-02-07/docs/1301.pdf.

17. Meadows, J. Gov. Rauner signs 'Gabby's Law' requiring sepsis protocols at all Illinois hospitals. Illinois Public Media (18 August 2016).

18. CDC. State policy approaches to sepsis prevention and early recognition. https:// www.cdc.gov/hai/pdfs/sepsis/vs-sepsis-policy-final.pdf (2021).

19. H.R.7514 - Gabby's Law Act. https://www.congress.gov/bill/116th-congress/housebill/7514/text? $r=3 \& s=1$

20. Davis, A. L. et al. American College of Critical Care Medicine clinical practice parameters for hemodynamic support of pediatric and neonatal septic shock. Crit. Care Med. 45, 1061-1093 (2017).

21. Weiss, S. L. et al. Surviving Sepsis Campaign International Guidelines for the management of septic shock and sepsis-associated organ dysfunction in children. Pediatr. Crit. Care Med. 21, e52-e106 (2020).

22. Lane, R. D., Funai, T., Reeder, R. \& Larsen, G. Y. High reliability pediatric septic shock quality improvement initiative and decreasing mortality. Pediatrics 138, e20154153 (2016).

23. Larsen, G. Y., Mecham, N. \& Greenberg, R. An emergency department septic shock protocol and care guideline for children initiated at triage. Pediatrics 127, e1585-e1592 (2011).

24. Paul, R. et al. A quality improvement collaborative for pediatric sepsis. Pediatr. Qual. Saf. https://doi.org/10.1097/pq9.0000000000000051 (2018).

25. Goldstein, B., Giroir, B. \& Randolph, A. International Pediatric Sepsis Consensus Conference: definitions for sepsis and organ dysfunction in pediatrics. Pediatr. Crit. Care Med. 6, 2-8 (2005).

26. Scott, H. F. et al. Development and validation of a predictive model of the risk of pediatric septic shock using data known at the time of hospital arrival. J. Pediatr. 217, 145.e6-151.e6 (2020).

27. Lloyd, J. K., Ahrens, E. A., Clark, D., Dachenhaus, T. \& Nuss, K. E. Automating a manual sepsis screening tool in a pediatric emergency department. Appl. Clin. Inform. https://doi.org/10.1055/s-0038-1675211 (2018).

28. Cruz, A. T. et al. Test characteristics of an automated age- and temperature-adjusted tachycardia alert in pediatric septic shock. Pediatr. Emerg. Care 28, 889-894 (2012).

29. Depinet, H. et al. Pediatric Septic Shock Collaborative: description of early improvement. Pediatrics 142, 110 (2018).

30. Balamuth, F. et al. Comparison of two sepsis recognition methods in a pediatric emergency department. Acad. Emerg. Med. 22, 1298-1306 (2015).

31. Balamuth, F. et al. Improving recognition of pediatric severe sepsis in the emergency department: contributions of a vital sign-based electronic alert and bedside clinician identification. Ann. Emerg. Med. 70, 759.e2-768.e2 (2017).

32. Eisenberg, M. et al. Comparison of manual and automated sepsis screening tools in a pediatric emergency department. Pediatrics 147, e2020022590 (2021).

33. Bradshaw, C. et al. Implementation of an inpatient pediatric sepsis identification pathway. Pediatrics 137, e20144082 (2016).
34. Dewan, M. et al. Design, implementation, and validation of a pediatric ICU sepsis prediction tool as clinical decision support. Appl. Clin. Inform. 11, 218-225 (2020).

35. Akcan Arikan, A. et al. Resuscitation bundle in pediatric shock decreases acute kidney injury and improves outcomes. J. Pediatr. 167, 1301.e1-1305.e1 (2015).

36. Balamuth, F. et al. Protocolized treatment is associated with decreased organ dysfunction in pediatric severe sepsis. Pediatr. Crit. Care Med. https://doi.org/ 10.1097/PCC.0000000000000858 (2016).

37. Eisenberg, M. et al. Outcomes of sepsis patients in a pediatric emergency department after automated sepsis screening. J. Pediatr. https://doi.org/10.1016/j. jpeds.2021.03.053 (2021).

38. Larsen, G. Y. et al. Development of a quality improvement learning collaborative to improve pediatric sepsis outcomes. Pediatrics 147, e20201434 (2021).

39. Bourgeois, F. T. \& Shannon, M. W. Emergency care for children in pediatric and general emergency departments. Pediatr. Emerg. Care 23, 94-102 (2007).

40. Gigli, K. H. et al. Pediatric outcomes after regulatory mandates for sepsis care. Pediatrics https://doi.org/10.1542/peds.2019-3353 (2020).

41. Schlapbach, L. J., Weiss, S. L. \& Wolf, J. Reducing collateral damage from mandates for time to antibiotics in pediatric sepsis-primum non nocere. JAMA Pediatr. https://doi.org/10.1001/jamapediatrics.2019.0174 (2019).

42. Mitka, M. Joint commission warns of alarm fatigue: multitude of alarms from monitoring devices problematic. JAMA 309, 2315-2316 (2013).

43. Kane-Gill, S. L. et al. Technologic Distractions (Part 1): summary of approaches to manage alert quantity with intent to reduce alert fatigue and suggestions for alert fatigue metrics. Crit. Care Med. 45, 1481-1488 (2017)

44. McGrath, S. P., Taenzer, A. H., Karon, N. \& Blike, G. Surveillance monitoring management for general care units: strategy, design, and implementation. Jt. Comm. J. Qual. Patient Saf. 42, 293-302 (2016).

45. Winters, B. D. et al. Technological Distractions (Part 2): a summary of approaches to manage clinical alarms with intent to reduce alarm fatigue. Crit. Care Med. 46, 130-137 (2018).

46. Austrian, J. S., Jamin, C. T., Doty, G. R. \& Blecker, S. Impact of an emergency department electronic sepsis surveillance system on patient mortality and length of stay. J. Am. Med. Inform. Assoc. https://doi.org/10.1093/jamia/ocx072 (2018).

47. Baker, A. H. et al. Effect of a sepsis screening algorithm on care of children with falsepositive sepsis alerts. J. Pediatr. https://doi.org/10.1016/j.jpeds.2020.12.034 (2020).

48. Singer, $M$. et al. The Third International Consensus Definitions for Sepsis and Septic Shock (Sepsis-3). JAMA 315, 801 (2016).

49. Delahanty, R. J., Alvarez, J. A., Flynn, L. M., Sherwin, R. L. \& Jones, S. S. Development and evaluation of a machine learning model for the early identification of patients at risk for sepsis. Ann. Emerg. Med. 73, 334-344 (2019).

50. Bedoya, A. D. et al. Machine learning for early detection of sepsis: an internal and temporal validation study. JAMIA Open 3, 252-260 (2020).

51. Le, S. et al. Pediatric severe sepsis prediction using machine learning. Front. Pediatr. 7, 413 (2019).

52. Phansalkar, S. et al. A review of human factors principles for the design and implementation of medication safety alerts in clinical information systems. J. Am. Med. Inform. Assoc. 17, 493-501 (2010).

53. Schubel, L. et al. Informatics and interaction: applying human factors principles to optimize the design of clinical decision support for sepsis. Health Inform. J. 26, 642-651 (2020)

\section{AUTHOR CONTRIBUTIONS}

M.A.E. and F.B. conceptualized the review, drafted the article, and gave final approval to the manuscript.

COMPETING INTERESTS

The authors declare no competing interests.

\section{ADDITIONAL INFORMATION}

Correspondence and requests for materials should be addressed to M.A.E.

Reprints and permission information is available at http://www.nature.com/ reprints

Publisher's note Springer Nature remains neutral with regard to jurisdictional claims in published maps and institutional affiliations. 